\title{
MAGNETO HYDRODYNAMIC GENERATOR
}

\author{
Rahul Rameshrao Parsodkar ${ }^{1 *}$ \\ *l Department of Power Electronics and Power System, Rashtrasant Tukadogi Maharaj Nagpur University Suresh \\ Deshmukh College of Engineering, Selukate, Wardha.rahul.parsodkar@gmail.com
}

*Corresponding Author: -

Email: rahul.parsodkar@gmail.com

\begin{abstract}
: -
Energy provides the power to progress. The natural resources of a country may be large but they can only be turned into wealth if they are developed, used and exchanged for other goods. This cannot be achieved without energy. Availability of sufficient energy and its proper use in any country can result in its people rising from subsistence level to the highest standards of living. It is interesting to note that more than half the population lives in Asia where the energy consumption is barely 8-10\% of the world's total. (More than half the world's primary energy consumed in North America and Western Europe.) Apart from the conventional energy sources of production of electricity which are Hydro, Thermal, and Nuclear, technological advances in progress for generation of electricity by direct means, i.e., without rotating parts such as 'Magneto Hydro Dynamics (MHD)'. Direct conversion of heat into electrical energy by means of MHD converters has not yet made its technological break-through though Magneto Hydro Dynamic generators are being actively examined in laboratories throughout the world. In India considerable studies have been carried out in this field by a team of scientist under the National Council of Science and Technology (NCST). It has been proposed to evolve a pilot MHD generation plant during the next few years in the country. The basic principle of MHD generation is the same as that of a conventional electrical generator, namely that the motion of a conductor through a magnetic field induces a voltage in the conductor. In MHD method gasses at high temperature are passed at high velocity through a magnetic field at right angles to the direction of flow and electricity is collected at stationary electrodes placed on opposite sides of the channel. This type of generation would greatly simplify the equipment and is likely to be developed in the next two decades.
\end{abstract}

Keywords: - MHD Generator, plasma, Faraday \& Lorentz Law, Ionization of gas, Fleming's Right hand rule .

\section{(c) $(\$)$}




\section{INTRODUCTION}

The MHD (magnetohydrodynamic) generator or dynamo transforms thermal energy or kinetic energy kinetic energy directly into electricity. MHD generators are different from traditional electric generators in that they can operate at high temperatures without moving parts moving parts. MHD was developed because the exhaust of a plasma MHD generator is a flame, still able to heat the boilers of a steam power plant. So high-temperature MHD was developed as a topping cycle to increase the efficiency of electric generation, especially when burning coal or natural gas. MHD dynamos are the complement of MHD propulsions, which have been applied to pump liquid metals and in several experimental ship engines. The interaction of moving conducting fluids with electric and magnetic fields provides for a rich variety of phenomena associated with electro-fluid-mechanical energy conversion. The field of MHD was initiated by Hanne's Alfven, for which he received the Nobel Prize in Physics in 1970.

$80 \%$ of total electricity produced in the world is hydal, while remaining $20 \%$ is produced from nuclear, thermal, solar, geothermal energy and from magneto hydro dynamic (MHD) generator.

MHD power generation is a new system of electric power generation which is said to be of high efficiency and low pollution. In advanced countries MHD generators are widely used but in developing countries like INDIA, it is still under construction, this construction work in progress at TRICHI in TAMIL NADU, under the joint efforts of BARC (Bhabha atomic research center), associated cement corporation (ACC) and Russian technologists. As its name implies magneto hydro dynamics (MHD) is concerned with the flow of a conducting fluid in the presence of magnetic and electric field. The fluid may be gas at elevated temperatures or liquid metals like sodium or potassium. Practical MHD devices have been in use since the early part of the 20th century. For example, an MHD pump prototype was built as early as 1907. More recently, MHD devices have been used for stirring, levitating, and otherwise controlling flows of liquid metals for metallurgical processing and other applications. Gas phase MHD is probably best known in MHD power generation. Since 1959, major efforts have been carried out around the world to develop this technology in order to improve electric conversion efficiency, increase reliability by eliminating moving parts, and reduce emissions from coal and gas plants.

\section{A. Structure of MHD Generator}

The simple magnetohydrodynamic generator consists of a gas nozzle. The gas nozzle is a combustion chamber that injects a pulse of gas into the channel/duct. The walls of the channel act as an electrode. The induced electric current is fed to the load by an external circuit that supplies the generated electricity to the desired destination. The MHD generators can be constructed in various designs like the Faraday generator, Hall generator and disc generator. Faraday generator was the first designed MHD generator. It was made by Michael Faraday in 1831. The Faraday generator used copper disks and a horse-shoe magnet to generate electricity. In the Faraday generator, a powerful electromagnet provides the magnetic field through which the plasma flows, and perpendicular to this field are installed the two electrodes on opposite sides of the plasma across which the electrical output voltage is generated. The current flowing across the plasma between these electrodes is called the Faraday current. This provides the main electrical output of the MHD generator. The basic concept underlying the mechanical and fluid dynamos is the same. The fluid dynamo, however, uses the motion of fluid or plasma to generate the currents which generate the electrical energy. The mechanical dynamo, in contrast, uses the motion of mechanical devices to accomplish this.

The functional difference between an MHD generator and an MHD dynamo is the path the charged particles follow. MHD generators are now practical for fossil fuels, but have been overtaken by other, less expensive technologies, such as combined cycles in which a gas turbine's or molten carbonate fuel cell's exhaust heats steam for steam turbine. Natural MHD dynamos are an active area of research in plasma physics and are of great interest to the geophysics and astrophysics communities. From their perspective the earth is a global MHD dynamo and with the aid of the particles on the solar wind produces the aurora borealis. The differently charged electromagnetic layers produced by the dynamo effect on the Earth's geomagnetic field enable the appearance of the aurora borealis. As power is extracted from the plasma of the solar wind, the particles slow and are drawn down along the field lines in a brilliant display over the poles.

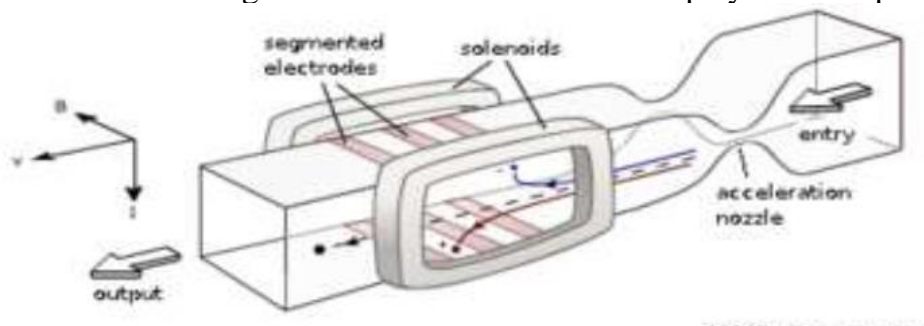

MHD Generator

Faraday linear nozzle with segmented olectrodes

\section{B. Working of MHD Generators}

The MHD generator can be considered to be a fluid dynamo. This is similar to a mechanical dynamo in which the motion of a metal conductor through a magnetic field creates a current in the conductor except that in the MHD generator the metal conductor is replaced by conducting gas plasma. It used for the generation of electrical energy. In the MHD generators, electricity generation is carried out using conducting fluid as an electrical conductor. The MHD is the acronym for magnetohydrodynamic generator. MHD generators are similar to the conventional electric generators. The only 
difference is that they use electrically conducting fluid instead of solid the Faraday current. This provides the main electrical output of the MHD generator. The basic concept underlying the mechanical and fluid dynamos is the same. The fluid dynamo, however, uses the motion of fluid or plasma to generate the currents which generate the electrical energy. The mechanical dynamo, in contrast, uses the motion of mechanical devices to accomplish this

\section{III.WORKING PRINCIPLE}

The principle of MHD generation is simple, based on Faraday's law of electromagnetic induction, i.e., when an electric conductor moves across a magnetic field, an e.m.f is induced in it, which produces an electric current. The conductor need not be a solid- it may be a gas or liquid. This is the principle of the conventional generator also, where the conductors consist of copper strips. In a MHD generator the solid conductors are replaced by a gaseous conductor (high pressure, high temperature combustion gas), i.e., an ionized gas. If such gas is passed at high velocity through a powerful or strong magnetic field, i.e., suppose we have a charged particle (having charge q·) moving at a high velocity v. towards right and a perpendicular magnetic field is applied. A magnetic force (Lorenz Force F) - acts on the charged particle. As shown in the figure below the positive ions would be accelerated towards the upper.

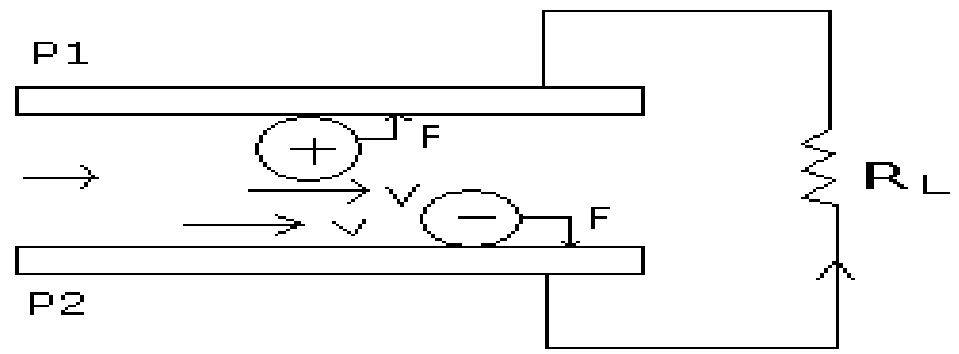

Consider, Plate P1 (cathode) and negative ions would be accelerated towards the lower plate P2 (anode). If the P1 and $\mathrm{P} 2$ are externally connected through the resistance, a current would flow through the resistance. Thus, gas energy is directly converted into electrical energy. This is the principle of MHD generator.

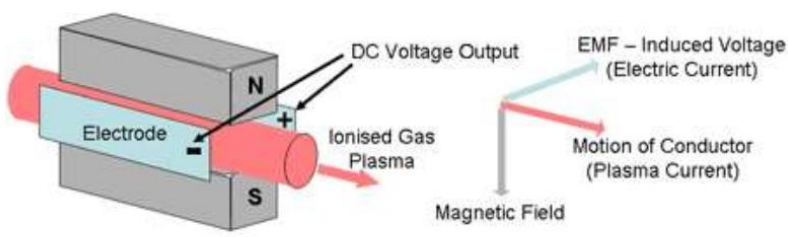

Magnetohydrodynamic Power Generation (Principle)

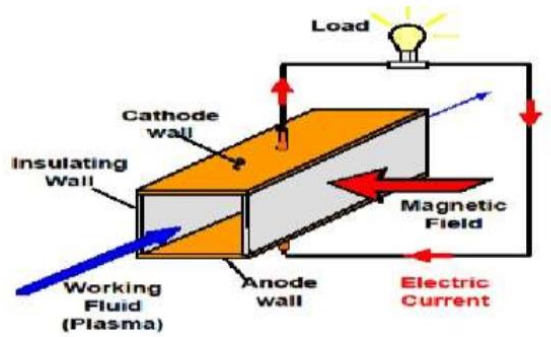

Lorentz Law describing the effects of a charged particle moving in a constant magnetic field can be stated as;

$\mathbf{F}=\mathbf{q} \cdot(\mathbf{v} \times \mathbf{B})$

Where,

F the force (Lorenz force) of the acting (Charged) particle

$\mathbf{v}$ the velocity of the particle (vector)

$\mathbf{q}$ the charge of the particle (scalar)

B the magnetic field (vector)

The vector $\mathrm{F}$ is perpendicular to both $\mathrm{v}$ and $\mathrm{B}$ according to Fleming's Right Hand Rule. 
When a conductor moves through a magnetic field it creates an electrical field perpendicular to the magnetic field and the direction of movement of the conductor. This is the principle, discovered by Michael Faraday, behind the conventional rotary electricity generator. Dutch physicist Anton Lorentz provided the mathematical theory to quantify its effects. The flow (motion) of the conducting plasma through a magnetic field causes a voltage to be generated (and an associated current to flow) across the plasma, perpendicular to both the plasma flow and the magnetic field according to 'Fleming's Right Hand Rule'.

Summarizing the above explanation, we can say that in a MHD system the kinetic energy of the working fluid is converted to electric energy. The figure given below shows a comparison between a turbogenerator and MHD generator.

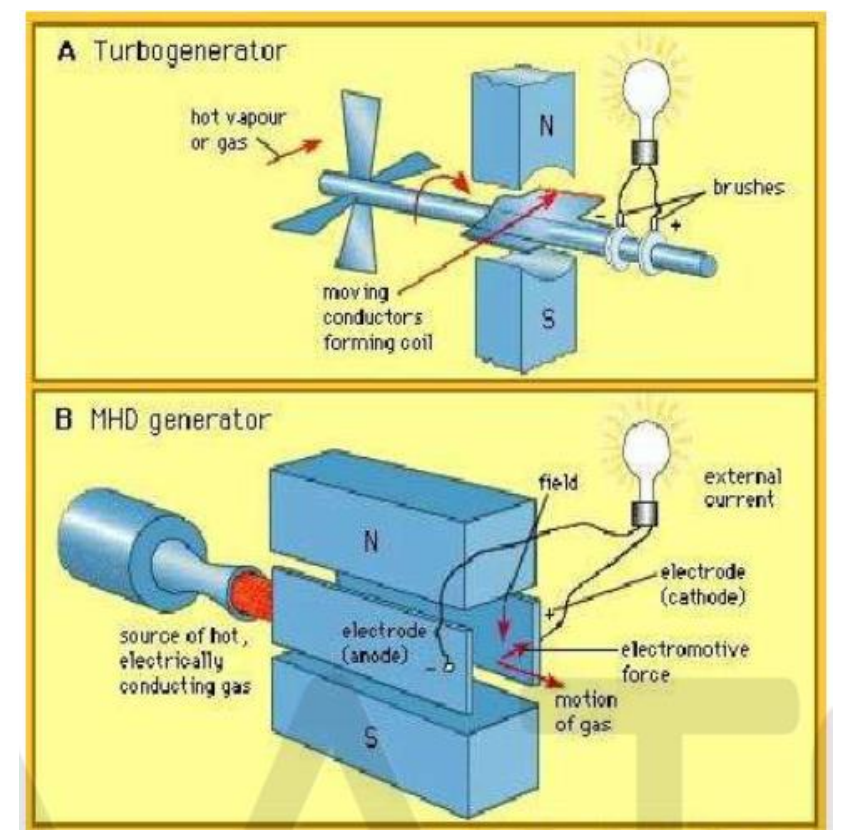

Here, in turbogenerator, the conductor moving inside the magnetic field is solid, while in MHD generator the conductor moving inside the magnetic field is in gaseous state. But both them on are working on the same principle, doing the same

work, giving the same output. However, the efficiency of both of them varies, as MHD generator gives much better and more output than the turbogenerator, hence it is more efficient. The MHD conversion is known as direct energy conversion because it produces electricity directly from heat source (derived from combustion of fuel) without the necessity of the additional stage of steam generation as in a steam power plant.

\section{THE MHD SYSTEM}

The MHD generator needs a high temperature gas source, which could be the coolant from a nuclear reactor or more likely high temperature combustion gases generated by burning fossil fuels, including coal, in a combustion chamber. The diagram below shows possible system components. The expansion no nozzle reduces the gas pressure and consequently increases the plasma speed through the generator duct to increase the power output. Unfortunately, at the same time, the pressure drop causes the plasma temperature to fall which also increases the plasma resistance. The exhaust heat from the working fluid is used to drive a compressor to increase the fuel combustion rate but much of the heat will be wasted unless it can be used in another process.

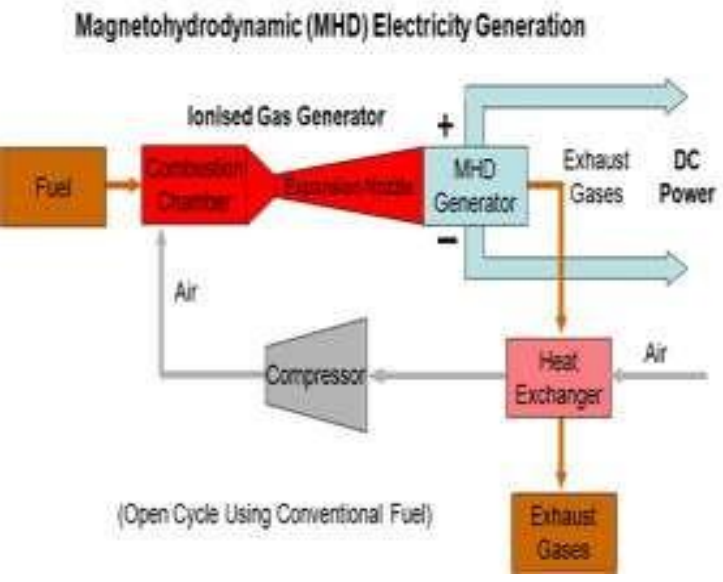




\section{A. The Plasma}

The Plasma in the MHD is created by a process called thermal ionization, where the temperature of the gas is raised to the point so that the electrons are no longer bound to the atoms of gas. These free electrons make the Plasma gas electrically conductive. The gas is continuously seeded with potassium nitrate or potassium carbonate, making the gas electrically conductive at lower temperature.

The prime system requirement is creating and managing the conducting gas plasma since the system depends on the plasma having a high electrical conductivity. Suitable working fluids are gases derived from combustion, noble gases, and alkali metal vapors. The gas plasma, to achieve high conductivity, the gas must be ionized, detaching the electrons from the atoms or molecules leaving positively charged ions of the gas. The plasma flows through the magnetic field at high speed, in some designs, more than the speed of sound, the flow of the charged particles providing the necessary moving electrical conductor.

\section{B. Methods of Ionizing the Gas}

Various methods for ionizing the gas are available, all of which depend on imparting sufficient energy to the gas. It may be accomplished by heating or irradiating the gas with X- rays or Gamma rays. It has also been proposed to use the coolant gases such as helium and carbon dioxide employed in some nuclear reactors as the plasma fuel for direct MHD electricity generation rather than extracting the heat energy of the gas through heat exchangers to raise steam to drive turbine generators. Seed materials such as Potassium carbonate or Cesium are often added in small amounts, typically about $1 \%$ of the total mass flow to increase the ionization and improve the conductivity, particularly of combustion gas plasmas.

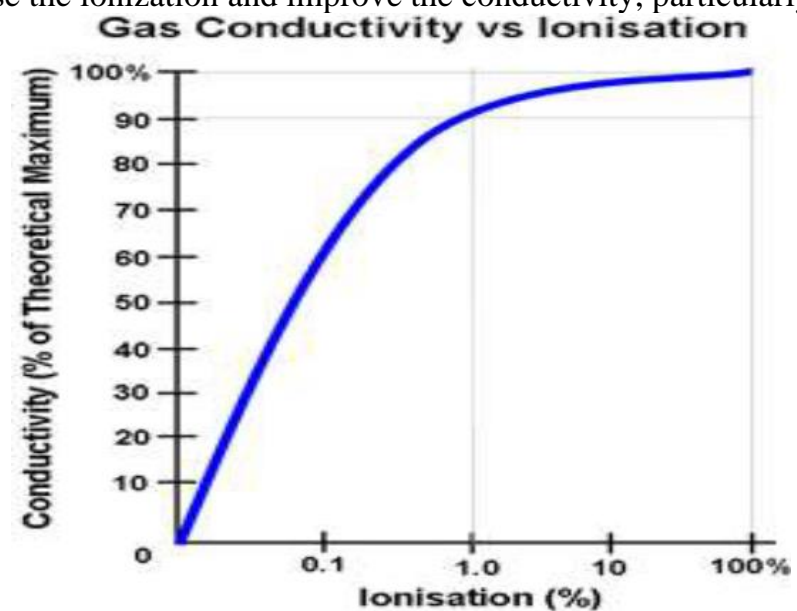

Note that on the logarithmic scale, the $90 \%$ conductivity can be achieved with a fairly low degree of ionization of only about $1 \%$.

\section{Power Output}

The output power is proportional to the cross-sectional area and the flow rate of the ionized plasma. The conductive substance is also cooled and slowed in this process. MHD generators typically reduce the temperature of the conductive substance from plasma temperatures to just over $1000{ }^{\circ} \mathrm{C}$. A MHD generator produces a direct current output which needs an expensive high-power inverter to convert the output into alternating current for connection to the grid.

\section{Efficiency}

Typical efficiencies of MHD generators are around 10 to 20 percent mainly due to the heat lost through the high temperature exhaust. This limits the MHD's potential applications as a stand-alone device but they were originally designed to be used in combination with other energy converters in hybrid applications where the output gases (flames) are used as the energy source to raise steam in a steam turbine plant. Total plant efficiencies of $65 \%$ could be possible in such arrangements.

\section{IV.ADVANTAGES OF MHD GENERATOR}

- Conventional coal-fired generators achieve a maximum efficiency of about 35\%. MHD generators have the potential to reach $50 \%$ - $60 \%$ efficiency.

- The higher efficiency is due to recycling the energy from the hot plasma gas to standard steam turbines. After the plasma gas passes through the MHD generator, it is still hot enough to boil water to drive steam turbines that produce additional power.MHD generators are also ecologically sound.

- Coal with high sulphur content can be used in the MHD without polluting the atmosphere. Although the cost cannot be predicted very accurately, yet it has been reported that capital costs of MHD plants will be competitive to conventional steam plants.

- It has been estimated that the overall operational costs in a plant would be about $20 \%$ less than conventional steam plants.

- Direct conversion of heat into electricity permits to eliminate the turbine (compared with a gas turbine power plant) or both the boiler and the turbine (compared with a steam power plant) elimination reduce losses of energy. 
- These systems permit better fuel utilization. The reduced fuel consumption would offer additional economic and special benefits and would also lead to conservation of energy resources.

- It is possible to use MHD for peak power generations and emergency service. It has been estimated that MHD equipment for such duties is simpler, has capability of generating in large units and has the ability to make rapid start to full load.

\section{A. Necessity of MHD System}

It is a well-known fact that at present a plenty of energy is needed to sustain industrial and agricultural production, and the existing conventional energy sources like coal, oil, uranium etc are not adequate to meet the ever-increasing energy demands. Consequently, sincere and untiring efforts have been made by scientists and engineers in exploring the possibilities of harnessing energy from several non-conventional energy sources. Magneto Hydro Dynamics (MHD) Generator is one of those energy sources. Today $80 \%$ of total electricity produced in the world is hydel, while remaining $20 \%$ is produced from nuclear, thermal, solar, geothermal energy and from magneto hydro dynamic power generation.

\section{B. Applications}

1) Power generation in space craft.

2) Hypersonic wind tunnel experiments.

3) Defense application.

4) Railway engines/Light weighted space vehicles.

The possibility to utilize MHD energy conversion systems in the space. We proposed a plasma MHD generator feed by fuel in liquid (Hydrogen, Oxygen, Nuclear fuel) or solid state.

The MHD generator proposed in built in the supersonic nozzle of a rocket engine utilizing liquid Hydrogen and Oxygen. The MHD interaction is effective in a layer near the wall at a temperature of about $2600 \mathrm{k}$ and at a pressure of 0.37 MPa. Hence, in order to increase the electrical conductivity, alkali metal (Potassium Carbonate or Cesium) seeding is used. We can also feed the small MHD generator to the railway engines for the increasing the better efficiency of railway engines, speed, and also increasing as well as controlling the energy.

\section{CONCLUSIONS}

With the increased industrial and agricultural activities, power demand is also highly increased. In the country is sure to fall short of the energy demand by the first decade of next century. This means an additional capacity of power is required next 10 year. The answer to this is in non- conventional energy. The MHD power generation is in advanced stage today and closer to commercial utilization significant progress has been made in development of all critical component and sub system technologies coal burning MHD combined steam power plant promise significant economic and environmental advantages compared to other coal burning power generate technologies. It will not be long before the technological problem of MHD generate are overcome and MHD power generation transform itself from nonconventional to conventional energy sources.

\section{REFERENCE}

[1].Non-Conventional energy source by G.D. Rai.

[2].Electrical Power System by Dr. Inamdar

[3].Generation Distribution \& Utilization of Electrical Energy by C.L.Wadhwa.

[4].A Textbook of Power System Engineering by R. K. Rajput

[5].S. Cuevas, S. Smolentsev, M. Abdou, Vorticity Generation in the Creeping Flow Past a Magnetic Obstacle, Physical Review E, in press, (2006).

[6].Kovalev , K.L.,Larionoff,A.E. and Plotavets, V.N.;"Theorotical and Experimental Studies of Faraday Multipol MHD Generators",Proc. 11Th Conf. on MHD Electrical Power Generation, Beijing,China, October(1992).

[7].Lineberry, J.T.et al.;”A Non-Equilibrium Plasma Generator”,Proc. 31 st Symp. Eng. Aspect MHD, VII.4 (1993). 